\title{
Butyrylcholinesterase deficiency
}

INSERM

\section{Source}

INSERM. (1999). Orphanet: an online rare disease and orphan drug data base.

Butyrylcholinesterase deficiency. ORPHA:132

Butyrylcholinesterase (BChE) deficiency is a metabolic disorder characterised by prolonged apnoea after the use of certain anaesthetic drugs, including the muscle relaxants succinylcholine or mivacurium and other ester local anaesthetics. The duration of the prolonged apnoea varies significantly depending on the extent of the enzyme deficiency. 